PUTP-95-23

\title{
Possible retardation effects of quark confinement on the meson spectrum
}

\author{
Cong-feng Qiao Han-Wen Huang Kuang-Ta Chao \\ Center of Theoretical Physics, CCAST(World Laboratory), Beijing 100080, P.R. China \\ Department of Physics, Peking University, Beijing 100871, P.R. China
}

\begin{abstract}
The reduced Bethe-Salpeter equation with scalar confinement and vector gluon exchange is applied to quark-antiquark bound states. The so called intrinsic flaw of Salpeter equation with static scalar confinement is investigated. The notorious problem of narrow level spacings is found to be remedied by taking into consideration the retardation effect of scalar confinement. Good fit for the mass spectrum of both heavy and light quarkomium states is then obtained.
\end{abstract}

PACS number(s): 


\section{Introduction}

To understand quark confinement is the most important task in QCD and hadron physics. Lattice QCD calculations show that the interquark potential for a heavy quarkantiquark pair $Q \bar{Q}$ in the static limit is well described by a linear confining potential, plus a short-ranged Coulomb potential[1]. Phenomenologically, these potentials have been used with the Schrödinger equation for nonrelativistic heavy quarkonium systems like $c \bar{c}$ and $b \bar{b}$ states, and satisfactory results for their mass spectrum have been obtained. Not only the spin-independent but also the spin-dependent $Q \bar{Q}$ potentials are studied both in lattice QCD [2] and the quark potential model[3, [4, 5]. Most results seem to be consistent with the picture that the dominant part of linear confinement potential is transformed as a Lorentz scalar, while the Coulomb potential stems from one gluon exchange, which has the feature of a Lorentz vector. In particular, the fact that the spin-orbit term (Thomas procession term) induced by the scalar confining potential tends to partially compensate the spin-orbit term generated by one gluon exchange, has been strongly supported by the observed fine splittings of P-wave $c \bar{c}$ and $b \bar{b}$ states [3, 国, 5.

However, a rather serious problem seems to remain if the spin-independent relativistic correction, caused by the static (instantaneous) scalar confining potential, is taken into consideration. This spin-independent term is of the same order as the Thomas precession term in the nonrelativistic expansion in terms of $\vec{p}^{2} / m^{2}$. As noted before[5], including this spin-independent term

$$
H_{S I}=-\frac{1}{4 m^{2}}\left(2 \vec{p}^{2} S+2 S \vec{p}^{2}+\frac{2}{r} \frac{d S}{d r}+\frac{d^{2} S}{d r^{2}}\right),
$$

where $S(r)$ is the static scalar confining potential and is usually assumed to take the form of $S(r)=\lambda r$ with $\lambda$ being the string tension, into the Hamiltonian will badly disturb the mass spectrum of mesons (even for $c \bar{c}$ states), because this term is negative and unreasonably large for higher excited states, making the level spacings for higher lying states unreasonably small. This problem is probably due to the fact that the scalar confining potential has been treated as an instantaneous potential, which is valid in the static limit but may not hold when relativistic corrections are taken into account. This problem was also noted by other authors [6] in the framework of the reduced Salpeter equation. This equation is equivalent to the Breit equation to the first order of $\vec{p}^{2} / \mathrm{m}^{2}$, and may be used to study higher order relativistic corrections for systems containing the charm quark and even lighter quarks. It was found [6] that in the framework of the reduced Salpeter equation with an instantaneous scalar confining potential, the level spacings (e.g.,the $2 S-1 S$ spacing) would tend to vanish for $q \bar{q}$ mesons when the constituent quark mass approachs to zero, and difficulties are already 
evident for the $c \bar{c}$ states. It was then pointed out 6 that there is an intrinsic flaw in the approach which uses the reduced Salpeter equation with static scalar confinement potential.

To overcome this difficulty, several scenarios have been put forward[6][7]. The chief differences between those works are of the useage of interaction potentials. By now, there are no mature theories or calculations for $q \bar{q}$ confinement interaction in QCD, and customarily used potentials are phenomenological and have some uncertain perameters in them. For different procedure of evaluating these parameters, one can have some different ways to fit the experimental data. Therefore, to classify some of the most effective alternatives of quark-antiquark interaction potentials seems still premature.

Despite of the limited understanding for confinement at present, more theoretical efforts should be made to study this problem. In our opinion, the difficulty with the reduced Salpeter equation and the static scalar confinement is probably due to the improper treatment that the confining interactiton is purely instantaneous. With some retardation effect of quark confinement being considered, even within the framework of reduced Salpeter equation the level spacings for $q \bar{q}$ mesons could become normal since the retardation effect might cancel the sick disturbance caused by the spin-independent correction from the instantaneous part of scalar confinement [8]. To implement this idea, we will assume the confinement kernel in momentum space to take the form

$$
G(q) \propto \frac{1}{\left(-q^{2}\right)^{2}}=\frac{1}{\left(\vec{q}^{2}-q_{0}^{2}\right)^{2}},
$$

where $q$ is the 4-momentum exchanged between the quark and antiquark in a meson. In fact, this form was suggested for the dressed gluon propagator at small momenta to implement confinement [9]. Here we will use the same form but regard it as an effective scalar confinement kernel. Then, if the system is not highly relativistic we may make the approximation

$$
G(q) \propto \frac{1}{\left(\vec{q}^{2}-q_{0}^{2}\right)^{2}} \approx \frac{1}{\left(\vec{q}^{2}\right)^{2}}\left(1+\frac{2 q_{0}^{2}}{\vec{q}^{2}}\right),
$$

and may further express $q_{0}$ in terms of its on-shell values which are obtained by assuming that quarks are on their mass shells. This should be a good approximation for $c \bar{c}$ and $b \bar{b}$ states, because they are nonrelativistic systems and the binding energies are smaller than the quark masses, therefore the quarks are nearly on their mass shells. In order to get a qualitative feeling about the retardation effect considered here, we will also use(3) for light quark mesons, though the approximations are not as good as for heavy quark mesons. With above approximations, the scalar confinement kernel becomes instantaneous again but we have incorperated some retardation effect into the kernel. In the static limit, the retardation term vanishes and the kernel returns to $G(q) \propto \frac{1}{\left(\vec{q}^{2}\right)^{2}}$, which is just the Fourier transform of the linear confining potential. 
In this paper, we will use this modified scalar confining potential in which the retardation effect is incorperated and the one-gluon-exchange potential in the framework of the reduced Salpeter equation to study the mass spectra of $q \bar{q}$ mesons including both heavy and light mesons. We will concentrate on the $0^{-}$and $1^{-}$mesons to examine their level spacings.

\section{Reduced Salpeter equation with scalar and vector interactions}

In quantum field theory, a basic description for the bound states is the Bethe-Salpeter equation 10]. Define the Bethe-Salpeter wave function of the bonud stase $|P\rangle$ of a quark $\psi\left(x_{1}\right)$ and an antiquark $\bar{\psi}\left(x_{2}\right)$ as

$$
\chi\left(x_{1}, x_{2}\right)=\left\langle 0\left|T \psi\left(x_{1}\right) \bar{\psi}\left(x_{2}\right)\right| P\right\rangle .
$$

where $T$ represents time-order product, and transform it into the momentum space

$$
\chi_{P}(q)=e^{-i P \cdot X} \int d^{4} x e^{-i q \cdot x} \chi\left(x_{1}, x_{2}\right) .
$$

Here $P$ is the four-momentum of the meson and $q$ is the relative momentum of quark and antiquark. We use the standard center of mass and relative variables:

$$
X=\eta_{1} x_{1}+\eta_{2} x_{2}, \quad x=x_{1}-x_{2}
$$

where $\eta_{i}=\frac{m_{i}}{m_{1}+m_{2}}(i=1,2)$. Then in momentum space the bound state BS equation reads

$$
\left(\not p_{1}-m_{1}\right) \chi_{P}(q)\left(\not p_{2}+m_{2}\right)=\frac{i}{2 \pi} \int d^{4} k G(P, q-k) \chi_{P}(k),
$$

where $p_{1}$ and $p_{2}$ represent the momentum of quark and antiquark respectively.

$$
p_{1}=\eta_{1} P+q, \quad p_{2}=\eta_{2} P-q .
$$

$G(P, q-k)$ is the interaction kernel which acts on $\chi$ and is determined by the interquark dynamics. Note in Eq.(7) $m_{1}$ and $m_{2}$ represent the effective constituent quark masses so that we could use the effective free propagators of quarks instead of the full propogators. This is an improtant approximation and simplification for light quarks. Furthermore, because of the lack of a fundamental description for the nonperturbative QCD dynamics, we have to make some approximations for the interaction kernel of quarks. In solving Eq.(77), we assume the kernel to be instantaneous (but with some retardation effect in a modified form for the kernel) and neglect the negative energy projectors in the quark propagators, because 
in general the negative energy projectors only contribute to quantities of higher orders due to $M-E_{1}-E_{2} \ll M+E_{1}+E_{2}$, where $\mathrm{M}, E_{1}$, and $E_{2}$ are the meson mass, the quark kinetic energy, and the antiquark kinetic energy respectively. Based on the above assumptions the BS equation can be reduced to a three-dimensional equation, i.e., the reduced Salpeter equation, for the three dimensional BS wave function

$$
\begin{gathered}
\Phi_{\vec{P}}(\vec{q})=\int d q^{0} \chi_{P}\left(q^{0}, \vec{q}\right) \\
\left(P^{0}-E_{1}-E_{2}\right) \Phi_{\vec{P}}(\vec{q})=\Lambda_{+}^{1} \gamma^{0} \int d^{3} k G(\vec{P}, \vec{q}, \vec{k}) \Phi_{\vec{P}}(\vec{k}) \gamma^{0} \Lambda_{-}^{2} .
\end{gathered}
$$

Here

$$
\begin{aligned}
& \Lambda_{+}^{1}=\frac{1}{2 E_{1}}\left(E_{1}+\gamma^{0} \vec{\gamma} \cdot \vec{p}_{1}+m_{1} \gamma^{0}\right), \\
& \Lambda_{-}^{2}=\frac{1}{2 E_{2}}\left(E_{2}-\gamma^{0} \vec{\gamma} \cdot \vec{p}_{2}-m_{2} \gamma^{0}\right),
\end{aligned}
$$

are the remaining positive energy projectors of the quark and antiquark respectively, and $E_{1}=\sqrt{m_{1}^{2}+{\overrightarrow{p_{1}}}^{2}}, E_{2}=\sqrt{m_{2}^{2}+{\overrightarrow{p_{2}}}^{2}}$. The formal products of $G \Phi$ in Eq.(10) take the form

$$
G \Phi=\sum_{i} G_{i} O_{i} \Phi O_{i}=G_{s} \Phi+\gamma_{\mu} \otimes \gamma^{\mu} G_{v} \Phi,
$$

where $O=\gamma_{\mu}$ corresponding to the pertubative one-gluon-exchange interaction and $O=1$ for the scalar confinement potential.

From Eq.(10) it is easy to see that

$$
\begin{aligned}
& \Lambda_{+}^{1} \Phi_{\vec{P}}(\vec{q})=\Phi_{\vec{P}}(\vec{q}), \\
& \Phi_{\vec{P}}(\vec{q}) \Lambda_{-}^{2}=\Phi_{\vec{P}}(\vec{q}) .
\end{aligned}
$$

Considering the constraint of Eq.(13), and the requirement of space reflection, in the rest frame of the meson $(\vec{P}=0)$ the wave function $\Phi_{\vec{P}}(\vec{q})$ for the $0^{-}$and $1^{-}$mesons can be written as

$$
\begin{aligned}
\Phi_{\vec{P}}^{0^{-}}(\vec{q}) & =\Lambda_{+}^{1} \gamma^{0}\left(1+\gamma^{0}\right) \gamma_{5} \gamma^{0} \Lambda_{-}^{2} \varphi(\vec{q}), \\
\Phi_{\vec{P}}^{-}(\vec{q}) & =\Lambda_{+}^{1} \gamma^{0}\left(1+\gamma^{0}\right) \not \gamma^{0} \Lambda_{-}^{2} f(\vec{q}),
\end{aligned}
$$

where $\phi=\gamma_{\mu} e^{\mu}, e^{\mu}$ is the polarization vector of $1^{-}$meson, and $\varphi(\vec{q}), f(\vec{q})$ are scalar functions of $\vec{q}^{2}$. It is easy to show that Eq.(14) is the most genernal form for the $0^{-}$and $1^{-}$(S-wave) 
$q_{1} \overline{q_{2}}$ meson wave functions at the rest frame [e.g., for the $0^{-}$meson wave function there are four independent scalar functions but with the constraint of Eq.(13) those scalar functions can be reduced to one and expressed exactly as Eq.(14)].

Substituting Eqs.(12) and (14) into Eq.(10), one derives the equations for $\varphi(\vec{q})$ and $f(\vec{q})$ in the meson rest frame [1]:

$$
\begin{aligned}
M \varphi_{1}(\vec{q})= & \left(E_{1}+E_{2}\right) \varphi_{1}(\vec{q}) \\
& -\frac{E_{1} E_{2}+m_{1} m_{2}+\vec{q}^{2}}{4 E_{1} E_{2}} \int d^{3} k\left(G_{S}(\vec{q}, \vec{k})-4 G_{V}(\vec{q}, \vec{k})\right) \varphi_{1}(\vec{k}) \\
& -\frac{\left(E_{1} m_{2}+E_{2} m_{1}\right)}{4 E_{1} E_{2}} \int d^{3} k\left(G_{S}(\vec{q}, \vec{k})+2 G_{V}(\vec{q}, \vec{k})\right) \frac{m_{1}+m_{2}}{E_{1}+E_{2}} \varphi_{1}(\vec{k}) \\
& +\frac{E_{1}+E_{2}}{4 E_{1} E_{2}} \int d^{3} k G_{S}(\vec{q}, \vec{k})(\vec{q} \cdot \vec{k}) \frac{m_{1}+m_{2}}{E_{1} m_{2}+E_{2} m_{1}} \varphi_{1}(\vec{k}) \\
& +\frac{m_{1}-m_{2}}{4 E_{1} E_{2}} \int d^{3} k\left(G_{S}(\vec{q}, \vec{k})+2 G_{V}(\vec{q}, \vec{k})\right)(\vec{q} \cdot \vec{k}) \frac{E_{1}-E_{2}}{E_{1} m_{2}+E_{2} m_{1}} \varphi_{1}(\vec{k}),
\end{aligned}
$$

where

$$
\begin{aligned}
& \varphi_{1}(\vec{q})=\frac{\left(m_{1}+m_{2}+E_{1}+E_{2}\right)\left(E_{1} m_{2}+E_{2} m_{1}\right)}{4 E_{1} E_{2}\left(m_{1}+m_{2}\right)} \varphi(\vec{q}) \\
M f_{1}(\vec{q})= & \left(E_{1}+E_{2}\right) f_{1}(\vec{q}) \\
& -\frac{1}{4 E_{1} E_{2}} \int d^{3} k\left(G_{S}(\vec{q}, \vec{k})-2 G_{V}(\vec{q}, \vec{k})\right)\left(E_{1} m_{2}+E_{2} m_{1}\right) f_{1}(\vec{k}) \\
& -\frac{E_{1}+E_{2}}{4 E_{1} E_{2}} \int d^{3} k G_{S}(\vec{q}, \vec{k}) \frac{E_{1} m_{2}+E_{2} m_{1}}{E_{1}+E_{2}} f_{1}(\vec{k}) \\
& +\frac{E_{1} E_{2}-m_{1} m_{2}+\vec{q}^{2}}{4 E_{1} E_{2} \vec{q}^{2}} \int d^{3} k\left(G_{S}(\vec{q}, \vec{k})+4 G_{V}(\vec{q}, \vec{k})\right)(\vec{q} \cdot \vec{k}) f_{1}(\vec{k}) \\
& -\frac{E_{1} m_{2}-E_{2} m_{1}}{4 E_{1} E_{2} \vec{q}^{2}} \int d^{3} k\left(G_{S}(\vec{q}, \vec{k})-2 G_{V}(\vec{q}, \vec{k})\right)(\vec{q} \cdot \vec{k}) \frac{E_{1}-E_{2}}{m_{2}+m_{1}} f_{1}(\vec{k}) \\
& -\frac{E_{1}+E_{2}-m_{2}-m_{1}}{2 E_{1} E_{2} \vec{q}^{2}} \int d^{3} k G_{S}(\vec{q}, \vec{k})(\vec{q} \cdot \vec{k})^{2} \frac{1}{E_{1}+E_{2}+m_{1}+m_{2}} f_{1}(\vec{k}) \\
& -\frac{m_{2}+m_{1}}{E_{1} E_{2} \vec{q}^{2}} \int d^{3} k G_{V}(\vec{q}, \vec{k})(\vec{q} \cdot \vec{k})^{2} \frac{1}{E_{1}+E_{2}+m_{1}+m_{2}} f_{1}(\vec{k})
\end{aligned}
$$

where

$$
f_{1}(\vec{q})=-\frac{m_{1}+m_{2}+E_{1}+E_{2}}{4 E_{1} E_{2}} f(\vec{q}) .
$$

Eqs. (15) and (17) can also be formally expressed as:

$$
\begin{aligned}
& \left(M-E_{1}-E_{2}\right) \varphi_{1}(\vec{q})=\int d^{3} k \sum_{i=S, V} F_{i}^{0^{-}}(\vec{q}, \vec{k}) G_{i}(\vec{q}, \vec{k}) \varphi_{1}(\vec{k}) \\
& \left(M-E_{1}-E_{2}\right) f_{1}(\vec{q})=\int d^{3} k \sum_{i=S, V} F_{i}^{1^{-}}(\vec{q}, \vec{k}) G_{i}(\vec{q}, \vec{k}) f_{1}(\vec{k})
\end{aligned}
$$


In most cases, the interaction kernel is of the convolution type, i.e., $G(\vec{q}, \vec{k})=G(\vec{q}-\vec{k})=$ $G(\vec{p})$, where $\vec{p}=\vec{q}-\vec{k}$ is the momentum exchanged between the quark and antiquark. In the nonrelativistic limit for both quark and antiquark, Eq. (15) and (17) can be expanded in

terms of $\vec{q}^{2} / m_{1}{ }^{2}$ and $\vec{q}^{2} / m_{2}{ }^{2}$, and they are identical with the Schrödinger equation to the zeroth order, and with the Breit equation to the first order.

\section{Interaction kernel and retardation for confinement}

To solve Eq.(77) one must have a good command of the potential between two quarks. At present, the reliable information about the potential only comes from the lattice QCD result, which shows that the potential for a heavy quark-antiquark pair $Q \bar{Q}$ in the static limit is well described by a long-ranged linear confining potential ( Lorentz scalar $V_{S}$ ) and a short-ranged one gluon exchange potential ( Lorentz vector $V_{V}$ ), i.e, [1, 2],

$$
V_{S}(\vec{r})=\lambda r, \quad V_{V}(\vec{r})=-\frac{4}{3} \frac{\alpha_{s}(r)}{r} .
$$

The lattice QCD result for the $Q \bar{Q}$ potential is supported by the heavy quarkonium spec-

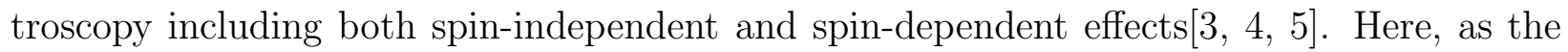
first step, we will employ the static potential below regardless of whether the quarks are heavy or not

$$
\begin{aligned}
& V(r)=V_{S}(r)+\gamma_{\mu} \otimes \gamma^{\mu} V_{V}(r), \\
& V_{S}(r)=\lambda r \frac{\left(1-e^{-\alpha r}\right)}{\alpha r}, \\
& V_{V}(r)=-\frac{4}{3} \frac{\alpha_{s}(r)}{r} e^{-\alpha r},
\end{aligned}
$$

where the introduction of the factor $e^{-\alpha r}$ is to avoid the infrared(IR) divergence and also to incorporate the color screening effects of the dynamical light quark pairs on the "quenched" $Q \bar{Q}$ potential [12]. It is clear that when $\alpha r \ll 1$ the potentials given in (21) become identical with that given in (20). In momentum space the potentials are

$$
\begin{aligned}
& G(\vec{p})=G_{S}(\vec{p})+\gamma_{\mu} \otimes \gamma^{\mu} G_{V}(\vec{p}), \\
& G_{S}(\vec{p})=-\frac{\lambda}{\alpha} \delta^{3}(\vec{p})+\frac{\lambda}{\pi^{2}} \frac{1}{\left(\vec{p}^{2}+\alpha^{2}\right)^{2}}, \\
& G_{V}(\vec{p})=-\frac{2}{3 \pi^{2}} \frac{\alpha_{s}(\vec{p})}{\vec{p}^{2}+\alpha^{2}}
\end{aligned}
$$


where $\alpha_{s}(\vec{p})$ is the well known running coupling constant and is assumed to become a constant of $O(1)$ as $\vec{p}^{2} \rightarrow 0$

$$
\alpha_{s}(\vec{p})=\frac{12 \pi}{27} \frac{1}{\ln \left(a+\frac{\vec{p}^{2}}{\Lambda_{Q C D}^{2}}\right)} .
$$

The constants $\lambda, \alpha, a$, and $\Lambda_{Q C D}$ are the parameters that characterize the potential.

Next, an important step is to take the retardation effect of scalar confinement into consideration. As disscussed in section I, the retardation effect of confinement will be approximatly treated by adding a retardation term $\frac{2 p_{0}^{2}}{\bar{p}^{6}}$ to the instantaneous part $\frac{1}{\left(\vec{p}^{2}\right)^{2}}$ as given in Eq.(3), and $p_{0}^{2}$ will be treated to take its on-shell values which are obtained by assuming that the quarks are on their mass shells. Then this retardation term will become instantaneous (but not convoluted). This modified scalar confinement potential will include the retardation effect and become

$$
\begin{aligned}
G_{S}(\vec{p}) \rightarrow G_{S}(\vec{p}, \vec{k}) & =-\frac{\lambda}{\alpha} \delta^{3}(\vec{p})+\frac{\lambda}{\pi^{2}} \frac{1}{\left(\vec{p}^{2}+\alpha^{2}\right)^{2}} \\
& +\frac{2 \lambda}{\pi^{2}} \frac{1}{\left(\vec{p}^{2}+\alpha^{2}\right)^{3}}\left(\sqrt{(\vec{p}+\vec{k})^{2}+m^{2}}-\sqrt{\vec{k}^{2}+m^{2}}\right)^{2} .
\end{aligned}
$$

This shows that the retardation effect of confinement is taken into consideration in the way that the interaction kernel depends not only on $\vec{p}$ (the momentum exchanged between quark and antiquark) but also on $\vec{k}$ (the momentum of the quark itself). By calculation below one may see that the retardation effect is not negligible for $c \bar{c}$ states and become very significant for light-quark systems and we find it might be an useful remedy for the "intrinsic flaw" of the reduced BS equation with static scalar confinement. In the computation in the next section we will use

$$
\lambda=0.183 \mathrm{GeV}^{2}, \quad \alpha=0.06 \mathrm{GeV}, \quad a=e=2.7183, \quad \Lambda_{Q C D}=0.15 \mathrm{GeV},
$$

All these numbers are in the scopes of customarily usage.

\section{Results and discussions}

Based on the formulism above, we have calculated the mass spectrum of quarkonium including both heavy- and light-quark systems. The numerical results with retardation are listed in Table I. The quark masses for the fit in Table I are

$$
m_{u}=0.35 \mathrm{GeV}, \quad m_{d}=0.35 \mathrm{GeV}, \quad m_{s}=0.5 \mathrm{GeV},
$$




$$
m_{c}=1.65 \mathrm{GeV}, \quad m_{b}=4.83 \mathrm{GeV} .
$$

\begin{tabular}{|c|c|c|c|c|c|c|}
\hline \multicolumn{7}{|c|}{$0^{-}$meson masses } \\
\hline & \multicolumn{2}{|c|}{$1 S$} & \multicolumn{2}{|c|}{$2 S$} & \multicolumn{2}{|c|}{$3 S$} \\
\hline States & $\begin{array}{c}\text { Fit } \\
(\mathrm{MeV})\end{array}$ & $\begin{array}{c}\text { Data } \\
(\mathrm{MeV})\end{array}$ & $\begin{array}{c}\text { Fit } \\
(\mathrm{MeV})\end{array}$ & $\begin{array}{c}\text { Data } \\
(\mathrm{MeV})\end{array}$ & $\begin{array}{c}\text { Fit } \\
(\mathrm{MeV})\end{array}$ & $\begin{array}{c}\text { Data } \\
(\mathrm{MeV})\end{array}$ \\
\hline$u \bar{u}, d d$ & 500 & $\pi(140)$ & 1252 & $\pi(1300)$ & 1611 & \\
\hline$s \bar{s}$ & 789 & & 1559 & & 1933 & \\
\hline$c \bar{c}$ & 2976 & $\eta_{c}(2980)$ & 3657 & & 4032 & \\
\hline$b \bar{b}$ & 9400 & & 9997 & & 10345 & \\
\hline \multicolumn{7}{|c|}{$1^{-}$meson masses } \\
\hline & \multicolumn{2}{|c|}{$\overline{1 S}$} & \multicolumn{2}{|c|}{$2 S$} & \multicolumn{2}{|c|}{$3 S$} \\
\hline States & $\begin{array}{c}\text { Fit } \\
(\mathrm{MeV})\end{array}$ & $\begin{array}{c}\text { Data } \\
(\mathrm{MeV})\end{array}$ & $\begin{array}{c}\text { Fit } \\
(\mathrm{MeV})\end{array}$ & $\begin{array}{c}\text { Data } \\
(\mathrm{MeV})\end{array}$ & $\begin{array}{c}\text { Fit } \\
(\mathrm{MeV})\end{array}$ & $\begin{array}{c}\text { Data } \\
(\mathrm{MeV})\end{array}$ \\
\hline$u \bar{u}, d d$ & 763 & $\omega(782)$ & 1359 & $\omega(1420)$ & 1673 & $\omega(1662)$ \\
\hline$s \bar{s}$ & 1025 & $\phi(1020)$ & 1649 & $\phi(1680)$ & 1989 & \\
\hline$c \bar{c}$ & 3119 & $J / \psi(3097)$ & 3701 & $\psi(3686)$ & 4062 & $\psi(4040)$ \\
\hline$b \bar{b}$ & 9460 & $\Upsilon(9460)$ & 10013 & $\Upsilon(10023)$ & 10353 & $\Upsilon(10355)$ \\
\hline
\end{tabular}

TABLE I. Calculated mass spectra of $b \bar{b}, c \bar{c}, s \bar{s}$, and $u \bar{u}$ or $d \bar{d}$ states using reduced Salpeter equation with retardation for scalar confinement. The Experiment data are taken from Ref. [13].

\begin{tabular}{|c|c|c|c|}
\hline Level Spacings & $\begin{array}{c}\text { Data } \\
(\mathrm{MeV})\end{array}$ & $\begin{array}{c}\text { With Retardation } \\
(\mathrm{MeV})\end{array}$ & $\begin{array}{c}\text { Without Retardation } \\
(\mathrm{MeV})\end{array}$ \\
\hline \multicolumn{4}{|c|}{$u \bar{u} d \bar{d}$ states } \\
\hline$\omega(2 S)-\omega(1 S)$ & 638 & 596 & 468 \\
$\omega(3 S)-\omega(1 S)$ & 880 & 910 & 727 \\
\hline \multicolumn{4}{|c|}{$s \bar{s}$ states } \\
\hline$\phi(2 S)-\phi(1 S)$ & 660 & 624 & 794 \\
$\phi(3 S)-\phi(1 S)$ & $?$ & 964 & 522 \\
\hline \multicolumn{4}{|c|}{$c \bar{c}$ states } \\
\hline$\psi(2 S)-J / \psi$ & 589 & 582 & 867 \\
$\psi(3 S)-J / \psi$ & 943 & 943 & 536 \\
\hline \multicolumn{4}{|c|}{$b b$ states } \\
\hline$\Upsilon(2 S)-\Upsilon(1 S)$ & 563 & 553 & 874 \\
$\Upsilon(3 S)-\Upsilon(1 S)$ & 895 & 893 & \begin{tabular}{c} 
B \\
\hline
\end{tabular}
\end{tabular}

TABLE II. The $2 S-1 S$ and $3 S-1 S$ energy level spacings of vector mesons with retardation and without retardation for scalar confinement

For comparison with the results obtained without retardation, in Table II we give a list of $2 S-1 S$ and $3 S-1 S$ energy level spacings for vector mesons in two cases, i.e., with retardation (using(24)) and without retardation (using (22)) for the scalar confinement potential. 
From Table I and Table II we can clearly see the following.

(1) The calculated level spacings without retardation are generally smaller than their experimental values. This trend is already appreciable for charmonium and becomes a serious problem for light quarkonium states. This result agrees with that obtained in Ref. [6]. We might improve the fit by readjusting the parameters (e.g., by enlarging the string tension), and this may work for low-lying heavy quarkonium states (e.g., $c \bar{c}$ states) but can not give a good global fit for high-lying states especially for light quarkonium states.

(2) By adding the retardation term to scalar confinement potential the calculated level spacings are significantly improved. The fit for $b \bar{b}$ and $c \bar{c}$ states is very good, and the fit for light vector mesons is also good, while the fit for light pseudoscalar mesons such as the pion is poor, which is probably due to the fact that the light pseudoscalar mesons are essentially Goldstone bosons and therefore the instantaneous and on-shell approximations no longer work well for them.

As emphasized in Section I, the approximate treatment for the retardation effect (in particular, the on-shell approximation) of scalar confinement should be good for heavy quarkonium states. Indeed, it has been shown 8 that for heavy quarkonium in nonrelativistic expansion the role of the retardation is just to cancel the troublesome term, $-\frac{1}{2 m^{2}}\left(\vec{p}^{2} S+S \vec{p}^{2}\right)$ in Eq.(四) and then remove the bad disturbance to the mass spectrum.

For light quarkonium states with constituent quark masses $m_{u}=m_{d} \approx 350 \mathrm{MeV}$, the retardation effect bocomes even more significant. In these systems nonrelativistic expansion is no longer good, but we can see the physical effect of retardation through an extreme case. That is the zero quark mass limit, which has been used for analysing the "intrinsic flaw" of scalar confinement, indicating that light quarks can only have very weak confinement if it is an instantaneous Lorentz scalar potential [6]. To see how the "retardation" part changes the trend of light quarks seeing a weaker confining potential than heavy quarks at large distances, it is useful to consider again the limit of zero quark mass. As light quark systems are more sensitive than heavy quark systems to the behavior of interaction at large distance, we will restrict our discussion only on the scalar confinement potential part.

In the zero-quark mass limit, as $m_{q} \rightarrow 0$, the coefficients for the scalar potential $G_{s}$ in Eq.(19) for the $0^{-}$and $1^{-}$mesons will reduce to

$$
\begin{aligned}
& F_{S}^{0^{-}}(\vec{q}, \vec{k}) \longrightarrow-\frac{1}{2}\left(1-\frac{\vec{q} \cdot \vec{k}}{q k}\right) \\
& F_{S}^{1^{-}}(\vec{q}, \vec{k}) \longrightarrow-\frac{1}{2} \frac{(\vec{q} \cdot \vec{k})}{q^{2}}\left(1-\frac{\vec{q} \cdot \vec{k}}{q k}\right) .
\end{aligned}
$$

where $q=|\vec{q}|, k=|\vec{k}|$. It is clear that these coefficients will vanish when $\vec{q} \rightarrow \vec{k}$. On 
the other hand, however, the static linear confining potential in momentum space behaves as $G_{s}(\vec{q}-\vec{k}) \propto(\vec{q}-\vec{k})^{-4}$ and is strongly weighted as $\vec{q} \rightarrow \vec{k}$ in Eq.(19). This is the reason why the light quarks can only have weak confinement, which leads to very narrow energy level spacings for light quarkonium states. This bad situation will be changed if the retardation is taken into account. In fact, the covariant form of confinement interaction may take the form $G_{S}(q, k) \propto\left[(\vec{q}-\vec{k})^{2}-\left(q_{0}-k_{0}\right)^{2}\right]^{-2}$ and in the on-shell approximation that $q_{0}^{2}=m^{2}+\vec{q}^{2}, k_{0}^{2}=m^{2}+\vec{k}^{2}$ it becomes

$$
G_{S}(q, k) \propto\left[-2 m^{2}+m^{2} \frac{k}{q}+m^{2} \frac{q}{k}+2(q k-\vec{q} \cdot \vec{k})\right]^{-2},
$$

where in the zero-quark mass limit $p, k \gg m \rightarrow 0$. We can see immediately that it has two distinct features from the static confining potential $G_{S}(\vec{q}, \vec{k}) \propto\left[(\vec{q}-\vec{k})^{2}\right]^{-2}$. First, with retardation the scalar interaction $G_{S}(q, k)$ is strongly weighted when $\vec{q}$ and $\vec{k}$ are co-linear $(\vec{q} \| \vec{k})$ that leads to $\vec{q} \cdot \vec{k}=q k$, whereas the static linear potential only peaks at $\vec{q}=\vec{k}$. This indicates that the former is strongly weighted in a much wider kinematic region than the latter. Second, when $\vec{q} \| \vec{k}, G_{S}(q, k) \propto O\left(m^{-4}\right)$, this mass dependence, which is absent in the static linear potential, will enhance confinement interaction and overwhelm the suppression factor apppearing in the coeficients $F_{S}^{0^{-}}$and $F_{S}^{1^{-}}$. As a result, even in the zero-quarkmass limit the effective scalar interaction will not be weakened, and this is just due to the retardation effect in the scalar interaction.

In practice, for the constituent quark model, which is essentially used in the present work, the quark mass can not be zero, and the on-shell approximation may not be as good as for heavy quark systems. But in any case the analysis given above in the zero mass limit is useful for understanding the qualitative feature of the retardation effect in quark confinement.

In this paper, we have tried to clarify the problem pointed out by Durand et al. for the static scalar confinement in reduced Salpeter equation. The "intrinsic flaw" of the Salpeter equation with static scalar confinement could be remedied to some extent by taking the retardation effect of the confinement into consideration. In the on-shell approximation for the retardation term of linear confinement, the notorious trend of narrow level spacings for quarkonium states especially for light quarkonium states is found to be removed. A good fit for mass spectrum of S-wave heavy and light quarkonium states (except the light pseudoscalar mesons) is obtained using one-gluon exchange potential and the scalar linear confinement potential with retardation taken into account. Although for light quark systems the on-shell appproximation may not be good, the qualitative feature of the retardation effect is still manifest. We may then conclude that at phenomenological level including the retardation effect into the scalar confinement may be necessary and significant. Nevertheless, it is still 
premature to assess whether or not quark confinement is really represented by the scalar

exchange of the form of $\left(\vec{p}^{2}-p_{0}^{2}\right)^{-2}$, as suggested by some authors as the dressed gluon propogator to implement quark confinement. We hope that our investigation can provide some useful information for the understanding of confinement. Further discussions concening heavy-light mesons will be given in another publication.

\section{Acknowlegement}

This work was supported in part by the National Natural Science Foundation of China, the State Education Commission of China and the State Commission of Science and Technology of China. 


\section{References}

[1] J.D. Stack, Phys. Rev. D29, 1213 (1984); S. Otto and J.D. Stack, Phys. Rev. Lett.52, 2328 (1984); D. Barkai et al., Phys. Rev. D 30, 1293 (1984).

[2] A. Huntley and C. Michael, Nucl. Phys. B286, 211 (1987); C. Michael, Phys. Rev. Lett.56, 1219(1986).

[3] For example, see: E. Eichten etal., Phys. Rev. D17, 3090 (1978); ibid. 21, 203 (1980); N. Byers and R.L. McClary, Phys. Rev. D28, 1692 (1983); A.B.Henriques, B.H. Kellett, and R.G. Moorhouse, Phys. Lett. 64B, 85(1976); P. Ditsas, N.A. MacDougall and R.G. Moorhouse, Nucl. Phys. B146, 191 (1978); D.P. Stanley and D. Robson, Phys. Rev. D21, 3180 (1980); S. Godfray and N. Isgur, ibid. 32, 189 (1985); S.N. Gupta, S.F. Radford and W.W. Repko ibid. 34, 201 (1986); M.G. Olsson and C.J. Suchyta, Phys. Rev. D36, 1459 (1987); L.J. Nickisch, L. Durand and B. Durand, Phys. Rev. D30, 660 (1984).

[4] E. Eichten and F. Feinberg, Phys. Rev. D23, 2734 (1981); D. Gromes, Z. Phys. C26, 401 (1984).

[5] Y.B. Ding, J. He, S.O. Cai, D.H. Qin and K.T. Chao, in Proceedings of International Symposium on Particles and Nuclear Physics, Beijing, China, 1985, edited by N. Hu ad C.-S. Wu (World Scientific, Singapore, 1987), p.88; Y.B. Ding, D.H. Qin and K.T. Chao, Phys. Rev. D44, 3652(1991).

[6] A. Gara, B. Durand, L. Durand and L.J. Nickisch, Phys. Rev. D40, 843 (1989); A. Gara, B. Durand and L. Durand, Phys. Rev. D42, 1651 (1990).

[7] see, for example, L.P. Fulcher, Z. Chen and K.C. Yeong, Phys. Rev. D47, 4122 (1993); L.P. Fulcher, Phys. Rev. D50, 447 (1994).

[8] K.T. Chao, PUTP-95-24.

[9] H. Pagels, Phy. Rev. D14, 2747(1976); Phys. Rev. D15, 2991(1977)

[10] E.E. Salpeter and H.A. Bethe, Phys. Rev. 84, 1232(1951); E.E. Salpeter, Phys. Rev. 87, 328(1952); D. Lurie, Particles and Fields (Wiley,1968); C. Itzykson and J.B. Zuber, Quantum Field Theory (McGraw-Hill,1980).

[11] K.T. Chao, talk given at the ITP Workshop on Hadron Transition Matrix Elements, Beijing, 1990(unpublished); J.H. Liu, Doctoral thesis, Peking University, 1993. 
[12] E. Laermann et al., Phys. Lett. B173, 437 (1986); K.D. Born, Phys. Rev. D40, 1653 (1989).

[13] Partical Data Group, L. Montanet etal., Phys. Rev. D50, 1173 (1994). 